First publ. in: Biological Psychology 60 (2002), pp. 17-36

\title{
High resolution EEG indicators of pain responses in relation to hypnotic susceptibility and suggestion
}

\author{
William J. Ray ${ }^{\mathrm{a}, *}$, Andreas Keil ${ }^{\mathrm{b}}$, Angelika Mikuteit ${ }^{\mathrm{b}}$, \\ Walter Bongartz ${ }^{\mathrm{b}}$, Thomas Elbert ${ }^{\mathrm{b}}$ \\ ${ }^{a}$ Department of Psychology, Penn State University, University Park, PA 16802, USA \\ ${ }^{\mathrm{b}}$ University of Konstanz, Konstanz, Germany
}

Received 2 November 2001; accepted 20 April 2002

\begin{abstract}
In this report we use a dense array (129 electrodes) EEG procedure to examine the effects of hypnotic susceptibility and hypnotic suggestions on electrocortical and self-report measures of painful stimuli. Self-report and event-related potential measures of six high and six low hypnotic susceptible individuals in response to pain were examined during an initial baseline condition and following a standard hypnotic induction under suggestions to either increase (hyperalgesia) or decrease (hypoalgesia) the painful stimulation. Our results show that high and low hypnotically susceptible individuals: (1) show few self-report or psychophysiological differences in response to baseline pain stimuli; (2) report differential pain experiences depending on hypnotic suggestions and (3) display differential psychophysiological indicators following an hypnotic induction with a suggestion of hypoalgesia. Overall, the findings suggest that hypnotic suggestions with high susceptible individuals modulate the later components of the evoked potential in a global manner and point up the importance of using both high and low hypnotically susceptible individuals preceding and following an hypnotic induction.
\end{abstract}

Keywords: Hypnotic susceptibility; Hyperalgesia; Hypoalgesia

\footnotetext{
* Corresponding author

E-mail address: wjr@psu.edu (W.J. Ray).
} 


\section{Introduction}

\subsection{Study of pain with brain imaging}

Pain has evolved as a critical process for the survival of the organism, which allows for immediate awareness concerning potential injury. The study of pain, however, has taken the field beyond a simple stimulus response sensory system into a complexity of systems involving at least affective evaluation, attention, cognitive appraisal and arousal systems (Kanda et al., 1996; Miron et al., 1989) which require the integrated activity of widely distributed networks that include cortical as well as subcortical structures. It has been suggested that in humans, besides sensory cortices, these structures may include the bilateral thalamus, the insula, the anterior cingulate cortex, and the premotor cortex (Casey, 1999) among others. Some of this evidence has been integrated in theoretical accounts of the hypothetical, parallel brain processes involved in pain (Melzack, 1999). However, these accounts need to be examined using methods such as high density event-related potential (ERP) studies capable of determining dynamic patterns of activity in terms of their spatial and temporal characteristics (Elbert and Keil, 2000).

With the advent of electrophysiological approaches (e.g. ERPs, EEG) and more recently brain imaging techniques (e.g. fMRI, PET, dense array EEG and MEG) it is now possible to monitor cerebral functioning in the human as related to pain (See Bromm and Lorenz, 1998 for a review). Using a variety of stimulation techniques, painful events have been shown to produce an early contralateral response in the primary somatosensory cortex (S1), followed by a more distributed contralateral activity in the $120-170 \mathrm{~ms}$ range that may also include ipsilateral components. Late responses display a bilateral pattern of activation. Since these later components are correlated with subjective reports of pain and only appear if the pain is felt, they are assumed to be related to cognitive or affective evaluation of the painful stimulus (Handwerker and Kobal, 1993). Anatomically, a variety of regions have been implicated in pain perception including primary and secondary somatosensory cortex, anterior cingulate cortex, basal ganglia and anterior frontal cortex (Lenz et al., 1998; Watanabe et al., 1998). Additionally, Ploghaus et al. (1999) were able to differentiate regions associated with the experience of pain (anterior cingulate, mid-insula, and anterior cerebellum) from those associated with the expectation of pain (anterior medial frontal cortex, anterior insula, and posterior cerebellum). In terms of mechanisms which modify the experience of pain, Yamasaki et al. (1999) found early event-related magnetic fields that showed a contralateral maximum (M1), and later evoked potentials maximal at vertex with a latency of 200-270 ms (N240) and 320-380 ms (P340) in response to painful laser stimulation. In this study distraction tasks did not affect the M1 components but did reduce the peak-to-peak amplitude of the EEG evoked potentials. Thus, a crucial question is the manner in which sensory and emotional aspects of pain can be modulated in a variety of situations. 


\subsection{Hypnotic modulation of pain}

One well documented behavioral intervention for the modulation of pain is that of hypnosis (See Chaves and Dworkin, 1997; Hilgard, 1973; Hilgard and Hilgard, 1994 and Holroyd, 1996 for reviews). Using hypnotic procedures, numerous clinical studies report reductions of pain under a variety of both chronic (e.g., cancer) and acute (e.g. painful medical procedures) conditions (Holroyd, 1996). Although hypnotic procedures have been documented for the past century, only outlines of a picture have emerged relating hypnosis to pain. A formal theoretical understanding is still lacking concerning the mechanisms by which pain reduction is accomplished as well as where in the chain of events hypnosis has an influence. At this point experimental studies suggest that hypnosis is different from relaxation, cognitive coping or a placebo-like mechanism not only with respect to subjective report but also with respect to behavioral and physiological patterns. Further, research with naloxone suggests that hypnotic influences on pain are not mediated by endogenous pain mechanisms involving endorphins (Spiegel and Albert, 1983). Thus, no clear model has emerged which describes the mechanisms by which hypnotic analgesia is accomplished.

\subsection{Psychophysiological studies of hypnotic analgesia}

Studies examining central nervous system processes during hypnotic analgesia have used a variety of paradigms including regional blood flow (Crawford et al., 1993), PET (Rainville et al., 1999), EEG (DePascalis et al., 1987), evoked potentials (Arendt-Nielsen et al., 1990), intracranial evoked potential (Kropotov et al., 1997). Overall, these studies report that hypnotic suggestions of analgesia can reduce both self-reports of painful experiences and modify physiological responses (see DePascalis, 2002 for an overview). A variety of studies have found that analgesia may be influenced by hypnotic susceptibility which has been shown to be a stable measure of individual differences with a test-retest correlation of at least 0.71 over 10, 15 , and 25 year periods (Piccione et al., 1989). For example, Crawford et al. (1993) found that during hypnotic analgesia only highly hypnotizable individuals showed differential blood flow changes. Likewise, DePascalis and Perrone (1996) found lower overall EEG amplitude for high hypnotizable individuals as compared to lows under either a no suggestion condition or a suggestion to reduce painful electrical stimulation. Other hypnotic analgesic studies (Arendt-Nielsen et al., 1990; Danziger et al., 1998; Rainville et al., 1999), although important, have limited their participants to high susceptible individuals making it more difficult to differentiate individual difference factors from those of hypnotic suggestions.

\subsection{Study goals}

In this present study, we are extending current work within this area of pain modulation using carefully screened high and low hypnotically susceptible individuals. We presented electroshocks to either the left or right finger under two different 
hypnotic suggestions (1) to dissociate (hypoalgesia) either the hand receiving the shock or the other one and (2) to sensitize (hyperalgesia) a particular hand for pain perception. In the study we examined (1) initial self-report and psychophysiological differences in relation to hypnotic susceptibility; and (2) differential pain experiences and electrocortical indicators of hypo- and hyper-algesia suggestions in relation to hypnotic susceptibility. In this report we describe the results using a dense array (129 electrodes) EEG procedure. In order to reduce the data available and to describe the topography of the ERPs, we utilize a procedure to determine the spatiotemporal structure of the signal as described in other studies (Dien et al., 1997; Spencer et al., 1999). Basically, a principal components analysis (PCA) was performed on the cortical source data in response to the pain stimuli to determine the spatial patterns in the data. This initial PCA allows electrodes that supply highly correlated information to be combined and thus creates a potentially smaller series of virtual electrodes. A second PCA was performed on these data to describe the temporal patterns present. The resulting spatiotemporal factor scores were used to describe the electrocortical activity in response to the pain stimuli.

\section{Methods}

\subsection{Participants}

Twelve university students ( 7 females, 5 males) with a mean age of 26 (range 21-32) years gave written informed consent to participate. These participants were selected from a larger pool of individuals on the basis of extreme scores on the Harvard group scale of hypnotic susceptibility, form B (Bongartz, in press; Shor and Orne, 1962) and the Stanford hypnotic susceptibility scale, form C (Bongartz, 2000; Weitzenhoffer and Hilgard, 1962). They were assigned to two groups: low-susceptible subjects $(N=6$; score $<3$ points $)$ and high-susceptible subjects $(N=6$; score $>8$ points). One subject was left-handed according to the Edingburgh Handedness Inventory (Oldfield, 1971). All subjects were paid for participation.

\subsection{Stimuli and procedure}

All participants went through two experimental sessions, 1 week apart. These sessions were identical except that EEG was recorded only in the second session and is the basis of this present report. The purpose of the first session was to make participants familiar with the experimental procedures. In both sessions, painful stimuli were electric shocks applied at the proximal joint of the middle finger using gold electrodes. Throughout the experiment, the electric stimuli were applied with a frequency of $1.004 \mathrm{~Hz}$ and duration of $10 \mathrm{~ms}$. Pain thresholds were determined for each middle finger using the method of limits. Using this method, the experimenter increased, then decreased, the current slowly. The subjects were asked first to indicate when they first experienced the electric pulse and second when they judged the sensation as slightly painful. The stimulus intensity was then further 
increased to $50 \%$ over the intensity that was felt as painful. The electric shock intensity then was continuously decreased, and the subjects were asked to indicate when the stimuli were no longer painful and when they didn't feel the pulse any longer. This procedure was repeated two times. For the threshold calculations only data from the final two series were used. Based on these calculations, stimulus intensities used during the EEG recordings were $50 \%$ higher than the individual pain thresholds.

Following threshold determinations, the participant sat in a comfortable chair in a small, darkened, noise-reduced room. A pre-hypnotic EEG-baseline of $45 \mathrm{~s}$ was recorded with painful electric stimulation for each hand. Subjects described the strength of their pain sensations after right and left stimulation using a $10-\mathrm{cm}$ visual analogue rating scale. Following the baseline, a hypnotic induction cassette was played $(8 \mathrm{~min})$ and was followed by one of 4 other $10 \mathrm{~min}$ instructional tapes. The suggestions on the four tapes were as follows: dissociation of the right hand, dissociation of the left hand, sensibilization of the right hand and sensibilization of the left hand. All participants received all four tapes with the order of presentation being counterbalanced over the participants. Following each tape, 90 pain stimuli were presented over a $90 \mathrm{~s}$ period resulting in 90 trials of EEG data. During the EEG recording, the participants fixated on a small yellow point approximately $1 \mathrm{~cm}$ in diameter. Participants also were instructed not to move their eyes or to blink. After each recording session, subjective pain experiences were rated.

\subsection{Data acquisition and analysis}

EEG was recorded from 129 electrodes using an $\mathrm{EGI}^{\mathrm{TM}}$ dense array with $\mathrm{Cz}$ being the reference electrode. Using standard procedures for the electrical geodesics high-input impedance amplifiers (Electrical Geodesics, 1998), impedances were kept below $50 \mathrm{k} \Omega$ (Ferree et al., 2001). Data were sampled at a rate of $200 \mathrm{~Hz}$ and an amplification factor of 10000 was used. An online band-pass filter ranging from 0.1 to $80 \mathrm{~Hz}$ was applied during recording. Further data processing and artifact rejection was performed offline using the procedure developed by Junghöfer et al. (2000). This procedure detects recording channel artifacts using the recording reference (i.e. Cz), converts data to average reference and detects global artifacts. It subsequently replaces sensors being contaminated with artifacts throughout with statistically weighted spherical spline interpolation from the full channel set, and computes the variance of the signal across trials to document the stability of the averaged waveform. Additionally, trials with ocular artifacts were omitted. Approximately 76 of the 90 trials in each condition were accepted. Statistical analysis demonstrated no significant difference in terms of condition or group in the trials retained. In this manner, artifact-free epochs containing $250 \mathrm{~ms}$ pre- and $800 \mathrm{~ms}$ post-stimulus were obtained and averaged according to the conditions of the study (pre-hypnosis, dissociation, and sensibilization for stimuli presented to each hand). 


\subsubsection{ERP global power measures}

As a measure of global cortical activity and for illustration of signal power across channels, we computed the global power $g(t)$ of the ERP signal. This metric reflects spatial variance, weighted with the variability of the ERP signal across trials. It was computed separately for the two groups and three experimental conditions according to Eq. (1):

$$
g(t)=\frac{\sum_{j=1}^{128} x_{j}(t)^{2} s_{j}(t)^{-1}}{128 \sum_{j=1}^{128} s_{j}(t)^{-1}}
$$

where $x_{j}(t)$, voltage at sensor $j$ and time $t$ and $s_{j}(t)$, standard deviation of voltage at sensor $j$ and time $t$ across trials.

\subsubsection{Source space analyses}

For estimation of cortical sources of ERPs, we used a minimum norm estimate (MNE; Hämäläinen and Ilmoniemi, 1984). The MNE is an inverse method for reconstructing the primary current that underlies an extracranially recorded brain potential. With the present algorithm, the origin of the signal is estimated using 655 model dipoles, being equidistantly arranged on three concentric shells (Hauk et al., 2002). The procedure is based on the assumption that the data vector $d$, which contains the recorded scalp potential at given electrode sites, can be described as the product of the leadfield matrix $L$, which specifies the electrode's sensitivity to the sources, the source current vector $j$ and a noise component $\varepsilon$ (see e.g. Grave de Peralta Menendez et al., 1997). Since $L$ and $d$ are known, and $\varepsilon$ is treated as if to be estimated with an acceptable accuracy, the MNE for $j$ can be determined as the mathematically unique solution of this equation which minimizes the squared current density $\left(j^{2}=\min \right)$. This solution is obtained by multiplying the pseudoinverse of the leadfield matrix $L$ with the data. A main property of the algorithm is the fact that locations that do not generate current that contributes to the measured scalp potential will have a zero dipole moment. Information about the number or locations of cortical sources is not required. A central issue concerning the accuracy of inverse solutions is the role of the depth of the sources. The present algorithm addresses this problem by computing a three-dimensional source space consisting of three concentric shells ( 80,60 , and $40 \%$ of electrode radius). Subsequently, an inverse solution is determined for these shells. These shells differ regarding their spatial resolution and depth sensitivity (Hauk et al., 2002). In particular, deeper shells are associated with less suppression of deep sources, but more blurring occurs. Regarding spatial resolution, the most superficial shell has the best spatial resolution, and the deepest shell has the worst, whereas the opposite is true for depth sensitivity. For this reason, we report the solutions for the shell at $60 \%$ electrode radius throughout. Simulations have shown that this shell yields the best signal-to-noise ratio for simulated dipolar sources across different depth and amplitude parameters (Hauk et al., 2002). For mapping of results and computation of dependent variables, we computed at each of the 129 electrode locations the 
magnitude of the MNE resulting from the square root of the sum of the squared dipole moments.

\subsubsection{Principal components analysis}

For the statistical analysis of the location and time course of components in source space, spatial and temporal PCA were applied to the MNE of all participants under all conditions, as has been suggested by recent work with dense array EEG/ERP measures (Spencer et al., 1999; Dien et al., 1997). Two steps were sequentially applied to the averaged data of all participants and conditions.

In a first step, spatial PCA of the covariance of MNE values was computed with electrode locations as variables, across time points for all subjects and conditions. Only the 160 time points representing the $800 \mathrm{~ms}$ post-stimulus period were included in this procedure. This step yielded 6 spatial factors (SFs) which together accounted for $97 \%$ of the variance. Based on the six SFs, weighted sums (i.e. the factor scores) of the 129 electrode sites were computed for all individual MNE data according to the factor loadings of each SF on each electrode site. Thus, time series of factor scores for each time point were generated, each time series reflecting the ERP portion being statistically related to a SF's specific topography (Spencer et al., 1999). These time series could be generated for each participant and condition.

In a second step, the covariance matrix of these factor scores was submitted to temporal PCA, having time points as observations. Five temporal factors resulted, which accounted for more than $1 \%$ of variance each (cumulative amount of variance: $98 \%$ ). Temporal factor scores for each SF were computed and represented a measure of source activity for a specific topography and time course.

\subsubsection{Statistical analysis}

Factor scores were evaluated by means of a 2 (high vs low hypnotic susceptibility) $\times 3$ (pre-hypnosis vs hyper- vs hypo-analgesia) $\times 2$ (left vs right pain stimulation) analysis of variance (ANOVA). Since the focus of the present study was on the effects of hypnotic analgesia/hyperalgesia, only those data for which sites of suggested analgesia/hyperalgesia and stimulation site were identical (i.e. right or left fingers) were included in these ANOVAs. Estimates for the effect size $\left(\eta^{2}\right)$ are reported for significant effects and interactions involving the group factor. Post-hoc tests were evaluated using Scheffé tests.

\section{Results}

\subsection{Self-report data}

In terms of main effects, the ANOVAs on self-report scores showed no significant main effect of group but a significant main effect for condition showing self-report score changes in response to the hypnotic manipulations $(F(2,20)=17.3, P<0.01)$. In addition, there was a condition by group interaction with the high susceptible 
individuals showing a greater spread of self-report scores in line with the hypnotic suggestions as compared to the low susceptible subjects $(F(2,20)=12.7, P<0.01$, $\eta^{2}=0.56$, see Fig. 1). Post-hoc testing revealed that this effect was due to increased pain ratings of the highs during the hyperalgesia suggestion $(P<0.01)$. Although in the expected direction, the self-report scores of the highs during the hypoalgesia suggestion were not significantly lower than pre-hypnosis scores. No significant effects of suggestion side or stimulation side were found.

\subsection{Electrophysiological data}

\subsubsection{Component structure of data}

As shown in the global power plot, reflecting the variance of the signal across electrodes (Fig. 2), two major deflections are visible in the global power of the grand mean ERPs. The first deflection was negative at central sites and showed its peak most pronounced at sites contralateral to the stimulation side, near $\mathrm{Cz}$, at latencies around 130-140 ms after administration of painful stimuli. This deflection may correspond to the N2 or N150 wave, which has often been observed in similar studies using painful transcutaneous electric stimulation. The second major deflection was negative at central sites, peaking at 270-300 ms post-stimulus. Waveforms for a subset of the 129-electrode array illustrate that central sites contributed most to the strength of the signal (Fig. 3).

Spatial PCA on MNE amplitudes revealed a first factor reflecting the stimulus artifact, with loadings showing a uniform distribution across sites (Fig. 4). Subsequent SFs showed a bilateral centro-anterior (SF 2) and a centro-posterior (SF 3) center of factor loadings. Spatial factor 4 (SF 4) displayed high loadings of right-hemispheric temporal sites, whereas SF 5 showed right-frontal and SF 6 fronto-central foci. Temporal PCA on MNE amplitude revealed a first factor (TF 1) with loadings peaking at $290 \mathrm{~ms}$ post-stimulus (Fig. 5). The second temporal

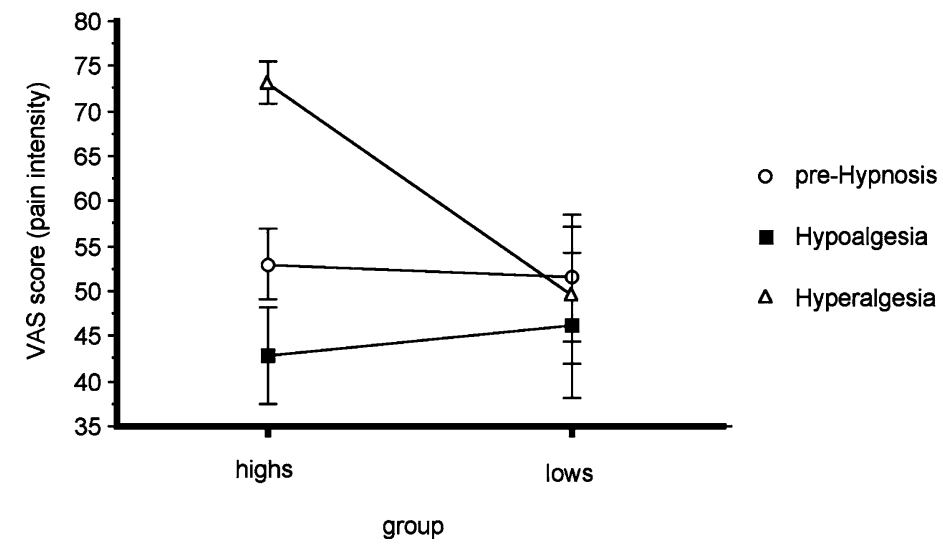

Fig. 1. Interaction plot of the group $\times$ suggestion effect on the visual analogue scale ratings of pain intensity. Values represent a mean of 6 subjects in each group. Bars indicate standard error. 

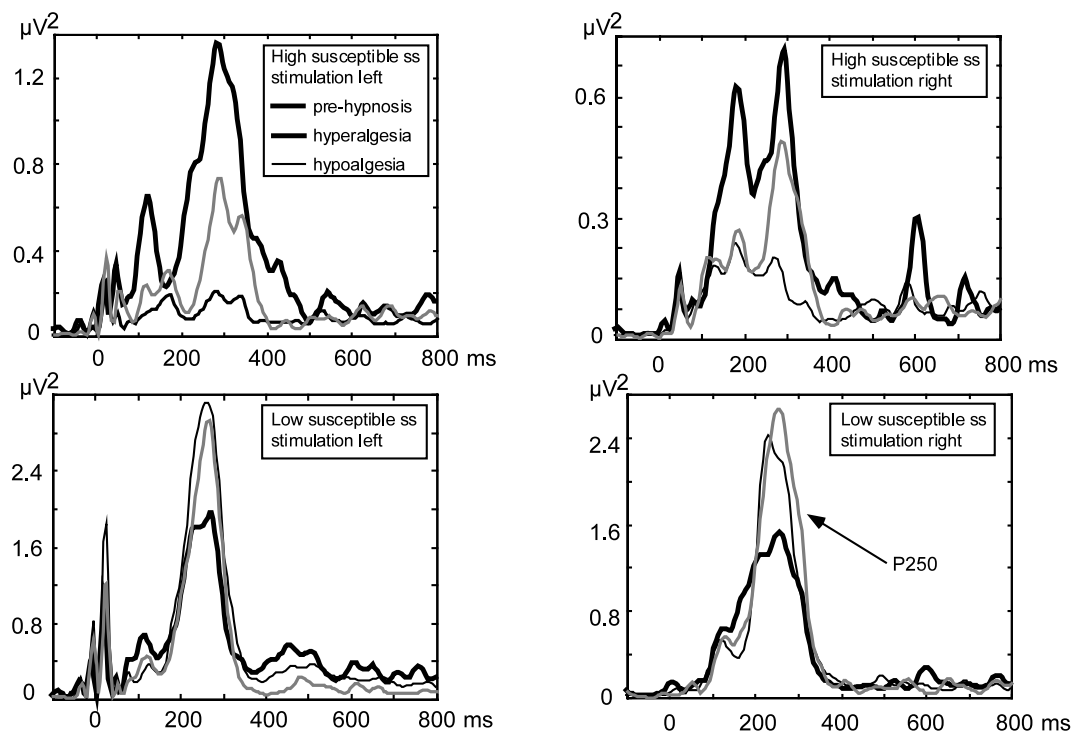

Fig. 2. Grand mean global power of ERPs under pre-hypnosis, hyperalgesia and hypoalgesia, obtained at 129 electrodes, computed by Eq. (1) (see Section 2). Left panels: ERPs obtained with stimulation at the left hand; right panels: ERPs obtained with stimulation at the right; top panels: High susceptible subjects; bottom panels: Low susceptible subjects. Note the stimulus artifact directly caused the electric stimulus applied to the left hand.

factor reflected the stimulus artifact, whereas loadings for the subsequent temporal factors peaked at $250 \mathrm{~ms}$ (TF 3), $140 \mathrm{~ms}$ (TF 4), and $340 \mathrm{~ms}$ (TF 5).

\subsection{ANOVAs on factor scores}

Given that the focus of the present study was on the variety of processes involved in the hypnotic modulation of pain perception, we did not restrict the statistical analyses to a subset of TFs and SFs. Thus, ANOVAs were performed for scores of the 4 temporal factors on $5 \mathrm{SFs}$ (excluding the factors representing the stimulus artifact respectively).

\subsubsection{Temporal factor 1 (N290)}

TF 1 factor scores showed no sensitivity for the group or hypnotic suggestion but rather displayed enhanced scores for left stimulation for SF $4(F(1,10)=18.4$, $P<0.01)$, SF $5(F(1,10)=25.4, P<0.01)$, and SF $6(F(1,10)=12.6, P<0.01)$.

\subsubsection{Temporal factor 3 (P250)}

The third temporal factor peaking at around $250 \mathrm{~ms}$ appeared to correspond to a positive deflection around $250 \mathrm{~ms}$ in the ERP that has often been reported in other work (e.g. Bromm and Scharein, 1982). The ANOVA on TF 3 scores for SF 2 showed a main effect of the group, with low suggestible participants exhibiting 

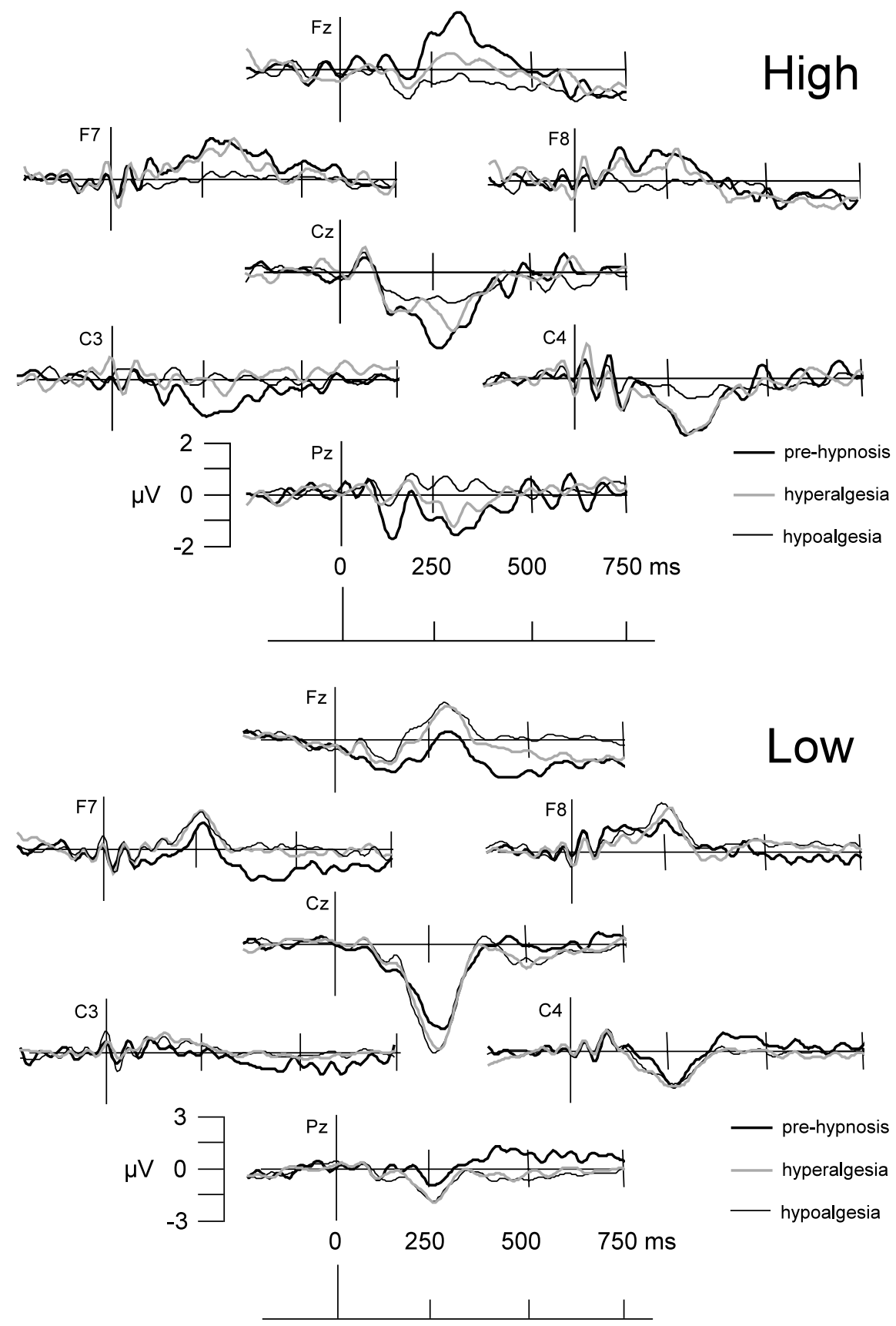

Fig. 3. Grand mean ERP waveforms at a subset of the electrode array. Top panel: high susceptible subjects $(N=6)$. Bottom panels: low susceptible subjects $(N=6)$. 


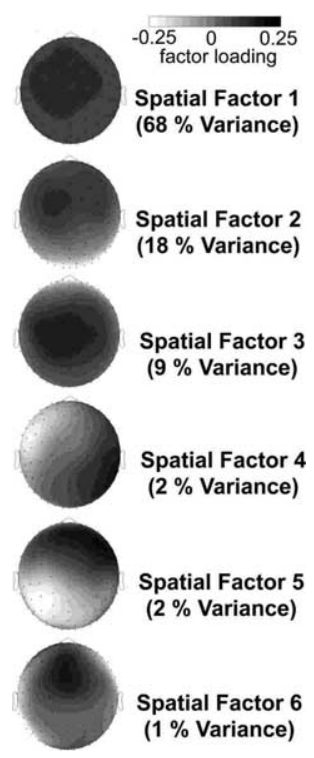

Fig. 4. Factor loadings derived from spatial PCA on MNE amplitudes, mapped at 129 sites corresponding to the sensor locations of the electrode montage (see Section 2 for explanation). Note that factor 1 reflects stimulus artifact.

higher scores than highs $(F(1,10)=5.0, P<0.05)$. Furthermore, an interaction (see Fig. 6) was found showing that the groups differentially reacted to hypnotic instruction (group $\times$ condition: $F(2,20)=4.7, P<0.05, \eta^{2}=0.33$ ). Post-hoc testing of this effect showed that groups did not differ in the pre-hypnosis condition, but differed during hypnotic induction, with highs exhibiting significantly decreased

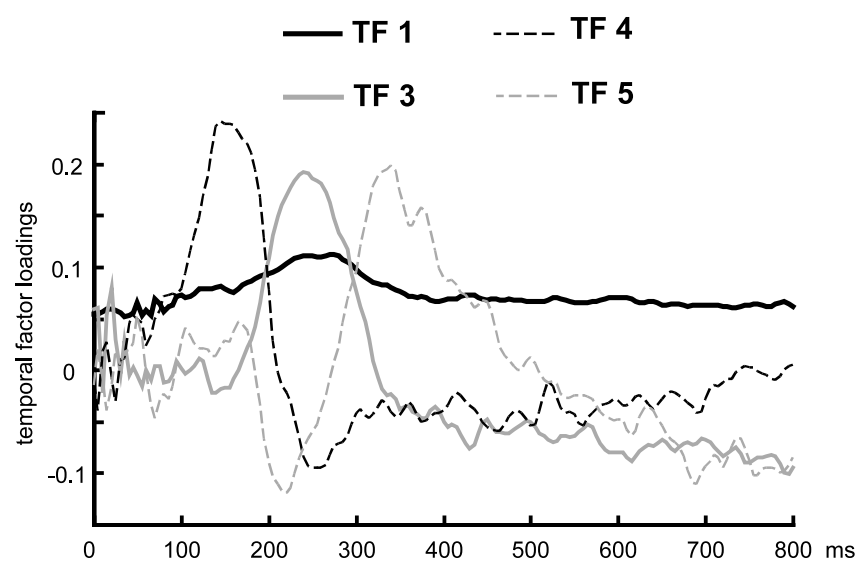

Fig. 5. Factor loadings derived from temporal PCA, which reflect temporal patterns present (see text for explanation). 
factor scores of TF 3 (P 250) on SF 2

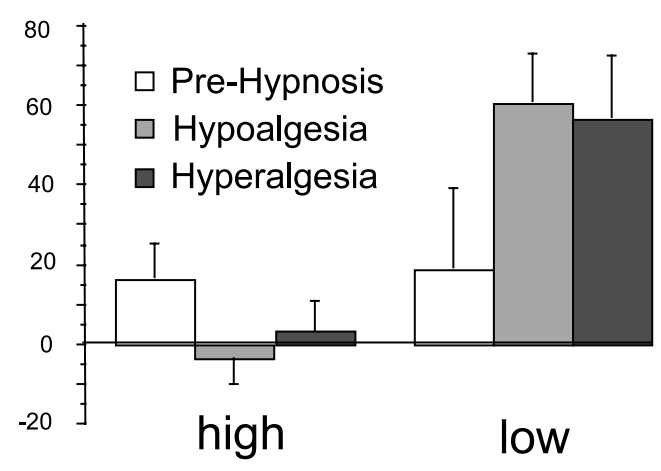

Fig. 6. Factor scores on temporal factor 3 using SF 2 for high and low hypnotically susceptible during baseline, hyperalgesia and hypoalgesia conditions. Bars indicate standard error.

scores during dissociation, whereas lows showed increased scores for both hypnotic conditions (i.e. hyper- and hypo-algesia). For SF 4, having a right temporal focus, symmetric responses to the lateralized stimuli were seen in the lows only whereas the highs displayed an asymmetric pattern with higher scores during left stimulation $\left(F(1,10)=5.8, P<0.05, \eta^{2}=0.37\right)$. A main effect of stimulation side across conditions and groups $(F(1,10)=7.2, P<0.05)$ was seen for scores on SF 5 (right-frontal focus of loadings). This indicated higher scores for stimulation on the left side without differentiating between groups and type of hypnotic suggestion. No significant effects of the P250 component were found for SF 6 (midline anterior focus of loadings).

\subsubsection{Temporal factor 4 (N140)}

Scores of TF 4 on SF 4 showed a main effect of stimulation side across conditions and groups $(F(1,10)=7.2, P<0.05)$, with higher scores being associated with ipsilateral (i.e. right) stimulation. Furthermore, N140 scores on SF 5 showed a significant interaction (group $\times$ condition $\times$ stimulation side: $F(2,20)=$ $4.9, P<0.05, \eta^{2}=0.3$ ). Post-hoc comparisons revealed that highs had significantly lower scores for both hypnotic conditions (analgesia and hyperalgesia suggestions) for left stimulation only, whereas right stimulation differences and differences between groups during pre-hypnotic stimulation did not reach significance. No further effect was found in this component.

\subsubsection{Temporal factor 5 (P340)}

The TF 5, with factor loadings peaking around $340 \mathrm{~ms}$, showed an effect of hypnotic suggestion for SF 6 only, across groups and stimulation sides $(F(1$, $10)=5.2, P<0.05)$. Follow-up testing of this effect showed that scores were significantly enhanced during pre-hypnosis across groups and stimulation sides compared with dissociation. 


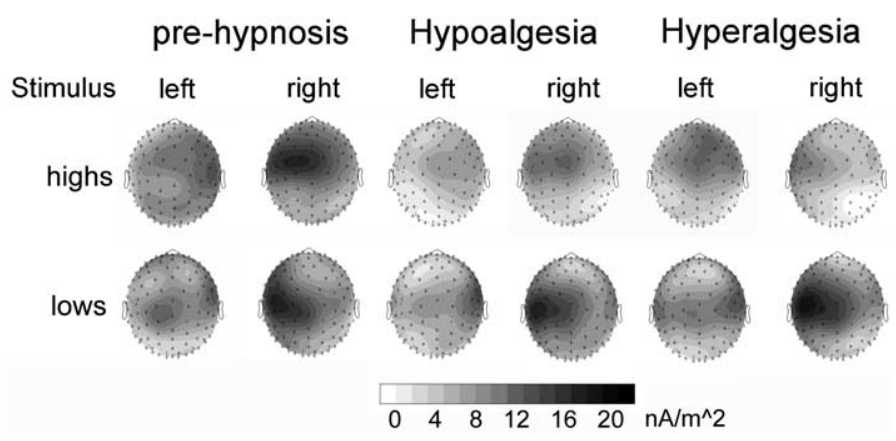

Fig. 7. Grand mean spline interpolated maps of the magnitude of the MNE for the N140 component (averaged MNE values for a 130-150 ms time window) under pre-hypnosis, hyperalgesia and hypoalgesia suggestions. Top row: high susceptible subjects; bottom row: low susceptible subjects.

\subsection{Source space analysis/MNE}

As expected, sources of the N140 component showed a pronounced lateralization to the hemisphere contralateral to the stimulus location. As shown in Fig. 7, this pattern was seen across conditions and groups. In addition, the unspecific decrease of dipole strength in the highs during hypnosis parallels the results of the scalp potential analysis. This further supports the notion that this early component is not differentially affected by the hypnotic suggestions. In terms of dipole orientations, we found that dipoles at sites near $\mathrm{Cz}$, where the dipole strength was greatest, were oriented tangentially from superior to inferior and from posterior to anterior. Dipole orientations did not show discrimination between groups or experimental conditions.

In contrast, the $\mathrm{P} 250$ showed a more central source configuration with a stronger modulation of dipole density as a function of hypnotic suggestion. In addition, the

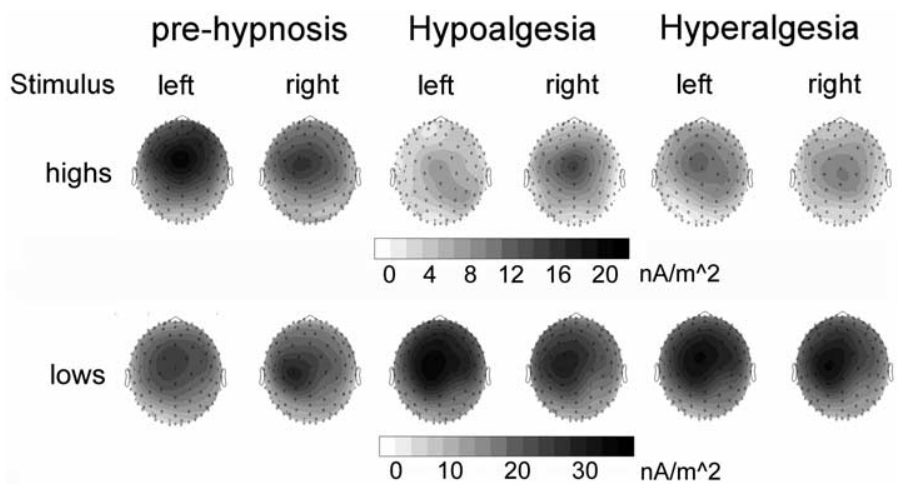

Fig. 8. Grand mean spline interpolated maps of the magnitude of the MNE for the P250 component (averaged MNE values for a 240-260 ms time window) under pre-hypnosis, hyperalgesia and hypoalgesia suggestions. Top row: high susceptible subjects; bottom row: low susceptible subjects. 
dipole strength of the MNE shown in Fig. 8 displayed a pattern of enhancement and reduction according to hypnotic suggestions, paralleling the findings in terms of scalp potential. Dipole orientations again did not differ in terms of groups and conditions, but showed radial orientations throughout in the regions with maximal dipole strength.

\section{Discussion}

In this report we describe a study using a dense array (129 electrodes) EEG procedure to examine the effects of hypnotic susceptibility and hypnotic suggestions on electro-cortical and self-report measures of painful stimuli. Self-report and electrocortical activity of high and low hypnotic susceptible individuals in response to pain was examined in an initial baseline condition and following a standard hypnotic induction under suggestions to either increase (hyperalgesia) or decrease (hypoalgesia) the painful stimulation. In this context we asked three specific questions: first, do high and low susceptible individuals respond differentially to the experience of pain; second, do hypnotic suggestions influence the experience of pain; and third, are there physiological mechanisms that differentially mediate the manner in which high and low susceptible individuals respond to these suggestions. We will first discuss our results in terms of specific analyses performed and then summarize these findings in terms of our three major questions.

\subsection{Self-report changes}

Self-report data displayed differential patterns of responding with low susceptible individuals showing little differentiation between the baseline, hypoalgesia, and hyperalgesia conditions whereas high susceptible individuals showed greater spread in the instructed direction. These results are consistent with a number of studies showing high susceptible individuals responsive to hypnotic suggestions (Danziger et al., 1998; Rainville et al., 1999). As seen in Fig. 1, the baseline self-report data show that high and low susceptible individuals do not differ in their ratings of the painful stimuli during the baseline, which suggests that high and low hypnotically susceptible people rate external stimuli in a similar way. This result gives support to the suggestion that any differential responding to the pain stimuli by the high and low susceptible participants were the influence of the hypnotic suggestions. In fact, consistent with previous reports, low susceptible individuals in our study did not respond to the hypnotic suggestions in the same manner as the high susceptible individuals. Their self-report data showed less variability between the baseline, hypo- and hyper-algesia conditions.

\subsection{Principal component analysis of the $M N E$}

In examining the overall pain-related evoked potential, the most striking feature is a drop in amplitude for the P250 (TF 1) component during the hypnosis 
condition for the high susceptible individuals and an increase in amplitude for the lows as compared to baseline. This was true for the SF showing high loading at central sites. The earlier N140 (TF 4) component, on the other hand, showed fewer and less specific changes during the hypnotic condition consistent with previous pain research showing these components to be more stimulus oriented (e.g. Gibson et al., 1991). In particular, this component was decreased non-specifically during both hypnosis conditions in the high susceptible subjects during left stimulation, at right temporal locations. The TF 1 (N290) did not show any sensitivity to group or condition at any of the SFs. Interestingly, the late P340 (TF 5) was modulated at prefrontal locations across groups showing a decrease for the dissociation condition, compared to pre-hypnosis. This effect was not specific to the highs, but was also seen in the lows. In terms of the later components, low susceptible individuals show an increase in power for both the hypo- and hyper-algesia conditions whereas high susceptible individuals display a decrease in power with the dissociation condition showing the greatest decrease. A variety of studies suggest that the later components are related to the painfulness of the stimuli and that these components may even be lacking when the stimuli are not experienced as painful (e.g. Gibson et al., 1991). These findings are consistent with our results in that during the dissociation condition the high susceptible individuals show smaller later components. This clearly suggests an inhibitory process for the high susceptible individuals associated with the hypnotic suggestions. This inhibitory process may be more of a global than specific nature since Spiegel et al. (1985) also reported reduced later ERP components when high hypnotic susceptible individuals were instructed to imagine stimuli on a computer screen to be blocked from vision. An intriguing possibility is that these inhibitory processes are related to the N290 (i.e. N2pb, $\mathrm{N} 2 \mathrm{pc}$ ) components described in terms of spatial visual attentional filtering (Luck and Hillyard, 1994; Wijers et al., 1997). Finally, given a variety of studies showing that the influence of cortical feedback on receptive field organization in areas such as the thalamus can be dramatically influenced by changes in higher order areas of the brain (Ergenzinger et al., 1998), it is possible for similar processes of the cortex to produce careful modulation of sensory experience such as that associated with the hypnotic modulation of pain.

In terms of the components found in the PCA of the overall MNE, the initial N140 component displayed a significant reduction in relation to the dissociation condition. Whether this is the result of a general relaxation condition following the hypnotic induction or a more specific influence cannot be determined from this study. The P250 component demonstrated a significant amplitude difference with low susceptible individuals showing larger amplitude response in comparison to the highs. Further, there was a significant interaction in which high susceptible individuals showed a significantly smaller response to the pain stimuli under the dissociation condition than during the pre-hypnosis baseline.

During pain reduction other authors also have found larger N2 and smaller P3 peaks for high hypnotic individuals although these studies did not use a PCA to determine the waveforms (e.g. DePascalis et al., 2001). In contrast, non-hypnotic studies generally report a P2 component to electroshock (Bromm and Lorenz, 
1998). In future research, there are a number of methodological considerations that should be addressed. First, the use of an average reference may give a different PCA outcome as compared to linked ear-lobe references. This is due to the fact that earlobe references apply differential weighting to the sensors, increasing the potential at central and midline sites and eventually neglecting activity at sites near the mastoids (Dien et al., 1997; Junghöfer et al., 1997). Since the P2 has been reported to have maximum amplitude at central midline sites, this could contribute to an overestimation of its size. Second, previous pain research has used a different mode of stimulation. We applied the electric pulses with constant strength and at constant intervals which could be associated with a decrease of the subjects' vigilance or arousal in the course of the experiment, especially since later positive ERP components have been associated with factors such as level of attention, arousal, and expectation (Gibson et al., 1991). Further research could clarify this question by the use of randomized stimulus intervals, varying stimulus intensities and an additional task to manipulate direction of attention (see Bromm and Lorenz, 1998 for recommendations).

Consistent with previous research the source space analysis of the N140 response showed a pronounced lateralization to the contralateral hemisphere from the side of the pain stimuli. This pattern was present not only in baseline but also in the hypnotic conditions in both groups. The P250 component on the other hand displayed a more central focus with a greater modulation of the dipole density as a function of hypnotic suggestion. This is consistent with the results of Kakigi et al. (1996), who also found negative components (N240 and N300) of evoked potentials maximal at vertex in response to laser stimulation which these researchers have seen as reflecting cingulate and subcortical areas. Our results from the distributed source modeling suggest that hypnotic suggestions in high susceptible individuals influence the somatosensory areas of the cortex. The view that the primary somatosensory cortex has a role in pain perception and is important for the discrimination and modulation of various aspects of pain has been supported by lesion, animal and human data (see Bushnell et al., 1999 for review). The mechanisms of this modulation may be varied, including inhibition of the sensory areas mediated by cortico-thalamic back-projections. Comparison of the early N140 and the later P250 in high and low susceptible subjects under pre-hypnosis and dissociation supports the idea that at earlier stages, the lateralized (i.e. contralateral) response is modulated by unspecific effects of the hypnosis procedure, showing no group $\times$ condition interaction. These early effects may be related to differences in arousal in the relevant circuitry that may be mediated by structures such as the parts of the ascending reticular arousal system (Robbins, 1997) or the intralaminar nuclei of the thalamus (Kinomura et al., 1996). Given the blocked design of the present study, it may be the case that the level of individual arousal was initially different in the hypnosis and pre-hypnosis conditions, thus causing different early processing of stimuli.

At the later stages, the high hypnotic susceptible individuals showed a significant decrease in the P250 amplitude in response to the dissociation suggestion whereas the low susceptible individuals displayed an increase during both the hypoalgesia 
and hyperalgesia conditions. The center of gravity of these later activations appeared to be at the central regions, showing a bilateral distribution. A variety of possible explanations may account for this result. For instance, affective evaluation of the incoming painful stimulus may be altered by the hypnosis procedure. Given that the SFs showing these effects for the P250 in our study show a wide distribution over central areas, it seems possible that brain areas that are activated as parts of the aversive response include the anterior cingulate or prefrontal cortex. Both structures are highly connected to subcortical structures known to be involved in defensive affective processing, such as the amygdaloid nuclei. Afferent modulation of somatosensory processing according to motivational or emotional stimulus parameters would parallel ERP findings from the visual system as reported by Cuthbert et al. (2000). An alternative explanation emphasizes that the present P250 modulation may reflect more specific cognitive processes that cause an inhibition of the pain-related cortical response and that follow the hypnotic suggestion. This would parallel ERP findings from selective attention in the visual or auditory modality (Hillyard and Anllo-Vento, 1998). In general, such attentional effects are assumed to be based on afferent activity of an attentional control network that includes deep structures such as the anterior cingulate (Carter et al., 1999), and posterior parietal as well as prefrontal neocortical areas (Corbetta, 1998; Desimone and Duncan, 1995). Future work may try to shed light on these questions by obtaining correlates of emotional state such as subjective ratings, skin conductance or facial EMG data, an approach recently initiated by DePascalis et al. (2001).

Concerning these later components, it should also be noted that although not statistically significant from baseline, the P250 amplitude in high hypnotically susceptible individuals decreased rather than increased which raises the possibility that hypnosis itself may activate inhibitory processes regardless of the direction of the hypnotic suggestion. This finding is also consistent with the regional cerebral blood flow studies of Rainville et al. (1997) in which hypnotic suggestions to increase or decrease pain were not found in the primary sensory regions but only the anterior cingulate. As suggested by Rainville et al. (1997), unintended attentional and habituation effects may be the reason that inhibition was observed when facilitation (i.e. increased sensitivity to pain) was expected. That is to say, during the sensitization condition, the participants would be more likely to attend to the pain than during the baseline condition, which in turn could lead to an habituation effect in which successive stimuli produce less of a physiological pain response over the course of the experiment. This in turn would lead to an overall decrease in grand average ERP power during the hyperalgesia condition.

\subsection{Overview and conclusions}

In summary, our present report suggests that high and low hypnotically susceptible individuals: (1) show few self-report or psychophysiological differences in response to baseline pain stimuli; (2) report differential pain experiences in response to hypnotic inductions and suggestions, and (3) display differential psychophysiological indicators following an hypnotic induction and hypo- and hyper-algesia 
suggestions. The findings suggest that hypnotic suggestions with high susceptible individuals modulate the later components of the evoked potential in a global manner. Further, our procedure illustrates the importance in pain research of using both high and low hypnotically susceptible individuals preceding and following an hypnotic induction. In this manner, future research could further differentiate the manner in which each group modifies the experience of pain as well as develop clinical procedures which maximize a given individual's modulation style.

\section{Acknowledgements}

The authors would like to thank Ursula Lommen for help in data acquisition. This work was supported by the Deutsche Forschungsgemeinschaft.

\section{References}

Arendt-Nielsen, L., Zachariae, R., Bjerring, P., 1990. Quantitative evaluation of hypnotically suggested hyperaesthesia and analgesia by painful laser stimulation. Pain 42 (2), 243-251.

Bongartz, W., Deutsche normen für die stanford hypnotic susceptibility scale: form B (SHSS: B), Experimentelle und Klinische Hypnose 15, in press.

Bongartz, W., 2000. Deutsche Normen für die Stanford Hypnotic Susceptibility Scale: Form C (SHSS: C). Experimentelle und Klinische Hypnose 16, 123-134.

Bromm, B., Scharein, E., 1982. Principal component analysis of pain-related cerebral potentials to mechanical and electrical stimulation in man. Electroencephalogr. Clin. Neurophysiol. 53, 94-103.

Bromm, B., Lorenz, J., 1998. Neurophysiological evaluation of pain. Electroencephalogr. Clin. Neurophysiol. 107 (4), 227-253.

Bushnell, M.C., Duncan, G.H., Hofbauer, R.K., Ha, B., Chen, J., Carrier, B., 1999. Pain perception: is there a role for primary somatosensory cortex? Proc. Natl. Acad. Sci. USA 96 (14), 7705-7709.

Carter, C.S., Botvinick, M.M., Cohen, J.D., 1999. The contribution of the anterior cingulate cortex to executive processes in cognition. Rev. Neurosci. 10 (1), 49-57.

Casey, K.L., 1999. Forebrain mechanisms of nociception and pain: analysis through imaging. Proc. Natl. Acad. Sci. USA 96 (14), 7668-7674.

Chaves, J., Dworkin, S., 1997. Hypnotic control of pain: historical perspectives and future prospects. Int. J. Clin. Exp. Hypnosis 45, 356-376.

Corbetta, M., 1998. Frontoparietal cortical networks for directing attention and the eye to visual locations: identical, independent, or overlapping neural systems? Proc. Natl. Acad. Sci. USA 95 (3), $831-838$.

Crawford, H.J., Gur, R.C., Skolnick, B., Gur, R.E., Benson, D.M., 1993. Effects of hypnosis on regional cerebral blood flow during ischemic pain with and without suggested hypnotic analgesia. Int. J. Psychophysiol. 15 (3), 181-195.

Cuthbert, B.N., Schupp, H.T., Bradley, M.M., Birbaumer, N., Lang, P.J., 2000. Brain potentials in affective picture processing: covariation with autonomic arousal and affective report. Biol. Psychol. 52 (2), 95-111.

Danziger, N., Fournier, E., Bouhassira, D., Michaud, D., De Broucker, T., Santarcangelo, E., Carli, G., Chertock, L., Willer, J.C., 1998. Different strategies of modulation can be operative during hypnotic analgesia: a neurophysiological study. Pain 75 (1), 85-92.

DePascalis, V., 2002. Hypnotic analgesia, obstructive attentional processes and psychophysiological mechanisms. Hypnosis International Monographs. M.E.G. Stiflung, Munich. 
DePascalis, V., Magurano, M., Bellusci, A., Chen, A., 2001. Somatosensory event-related potential and autonomic activity to varying pain reduction cognitive strategies in hypnosis. Clin. Neurophysiol. $112,1475-1485$.

DePascalis, V., Marucci, F.S., Penna, P.M., Pessa, E., 1987. Hemispheric activity of $40 \mathrm{~Hz}$ EEG during recall of emotional events: differences between low and high hypnotizables. Int. J. Psychophysiol. 5 (3), $167-180$.

DePascalis, V., Perrone, M., 1996. EEG asymmetry and heart rate during experience of hypnotic analgesia in high and low hypnotizables. Int. J. Psychophysiol. 23, 163-175.

Desimone, R., Duncan, J., 1995. Neural mechanisms of selective visual attention. Annu. Rev. Neurosci. $18,193-222$.

Dien, J., Tucker, D.M., Potts, G., Hartry-Speiser, A., 1997. Localization of auditory evoked potentials related to selective intermodal attention. J. Cog. Neurosci. 9, 799-823.

Elbert, T., Keil, A., 2000. Imaging in the fourth dimension. Nature 404 (6773), 29-31.

Electrical Geodesics, 1998. Net Amps User Manual Eugene, OR: Electrical Geodesics, Inc.

Ergenzinger, E.R., Glasier, M.M., Hahm, J.O., Pons, T.P., 1998. Cortically induced thalamic plasticity in the primate somatosensory system. Nat. Neurosci. 1 (3), 226-229.

Ferree, T., Luu, P., Russell, J., Tucker, D., 2001. Scalp-electrode impedance, infection risk and EEG data quality. Clin. Neurophysiol. 112, 536-544.

Gibson, S.J., LeVasseur, S.A., Helme, R.D., 1991. Cerebral event-related responses induced by $\mathrm{CO}_{2}$ laser stimulation in subjects suffering from cervico-brachial syndrome. Pain 47 (2), 173-182.

Grave de Peralta Menendez, R., Hauk, O., Gonzalez Andino, S., Vogt, H., Michel, C., 1997. Linear inverse solutions with optimal resolution kernels applied to electomagnetic tomography. Human Brain Mapping 5, 454-467.

Hämäläinen, M., Ilmoniemi, R., 1984. Interpreting measured magnetic fields of the brain: estimates of of current distributions. (Technical Report TKK-F-A559). Helsinki University of Technology, Helsinki.

Handwerker, H.O., Kobal, G., 1993. Psychophysiology of experimentally induced pain. Physiol. Rev. 73 (3), 639-671.

Hauk, O., Keil, A., Elbert, T., Müller, M.M., 2002. Comparison of data transformation procedures to enhance topographical accuracy in time series analysis of the human EEG. J. Neurosci. Meth. 113 (2), $111-122$.

Hilgard, E.R., 1973. A neodissociation interpretation of pain reduction in hypnosis. Psychol. Rev. 80, $396-411$.

Hilgard, E.R., Hilgard, J.R., 1994. Hypnosis in the Relief of Pain. Brunner/Mazel Inc, New York.

Hillyard, S.A., Anllo-Vento, L., 1998. Event-related brain potentials in the study of visual selective attention. Proc. Natl. Acad. Sci. USA 95 (3), 781-787.

Holroyd, J., 1996. Hypnosis treatment of clinical pain: understanding why hypnosis is useful. Int. J. Clin. Exp. Hypnosis 44, 33-51.

Junghöfer, M., Elbert, T., Leiderer, P., Berg, P., Rockstroh, B., 1997. Mapping EEG-potentials on the surface of the brain: a strategy for uncovering cortical sources. Brain Topogr. 9 (3), 203-217.

Junghöfer, M., Elbert, T., Tucker, D.M., Rockstroh, B., 2000. Statistical control of artifacts in dense array EEG/MEG studies. Psychophysiology 37 (4), 523-532.

Kakigi, R., Koyama, S., Hoshiyama, M., Kitamura, Y., Shimojo, M., Watanabe, S., 1996. Pain-related brain responses following $\mathrm{CO}_{2}$ laser stimulation: magnetoencephalographic studies. Electroencephalogr. Clin. Neurophysiol. Suppl. 47, 111-120.

Kanda, M., Fujiwara, N., Xu, X., Shindo, K., Nagamine, T., Ikeda, A., Shibasaki, H., 1996. Pain-related and cognitive components of somatosensory evoked potentials following $\mathrm{CO}_{2}$ laser stimulation in man. Electroencephalogr. Clin. Neurophysiol. 100 (2), 105-114.

Kinomura, S., Larsson, J., Gulyas, B., Roland, P.E., 1996. Activation by attention of the human reticular formation and thalamic intralaminar nuclei. Science 271 (5248), 512-515.

Kropotov, J.D., Crawford, H.J., Polyakov, Y.I., 1997. Somatosensory event-related potential changes to painful stimuli during hypnotic analgesia: anterior cingulate cortex and anterior temporal cortex intracranial recordings. Int. J. Psychophysiol. 27 (1), 1-8. 
Lenz, F.A., Rios, M., Zirh, A., Chau, D., Krauss, G., Lesser, R.P., 1998. Painful stimuli evoke potentials recorded over the human anterior cingulate gyrus. J. Neurophysiol. 79 (4), 2231-2234.

Luck, S.J., Hillyard, S.A., 1994. Spatial filtering during visual search: evidence from human electrophysiology. J. Exp. Psychol. Hum. Percept. Perform. 20 (5), 1000-1014.

Melzack, R., 1999. From the gate to the neuromatrix. Pain (Suppl. 6), S121-126.

Miron, D., Duncan, G.H., Bushnell, M.C., 1989. Effects of attention on the intensity and unpleasantness of thermal pain. Pain 39 (3), 345-352.

Oldfield, R.C., 1971. The assessment and analysis of handedness: the Edinburgh inventory. Neuropsychologia 9, 97-113.

Piccione, C., Hilgard, E.R., Zimbardo, P.G., 1989. On the degree of stability of measured hypnotizability over a 25-year period. J. Pers. Soc. Psychol. 56 (2), 289-295.

Ploghaus, A., Tracey, I., Gati, J.S., Clare, S., Menon, R.S., Matthews, P.M., Rawlins, J.N., 1999. Dissociating pain from its anticipation in the human brain. Science 284 (5422), 1979-1981.

Rainville, P., Duncan, G.H., Price, D.D., Carrier, B., Bushnell, M.C., 1997. Pain affect encoded in human anterior cingulate but not somatosensory cortex. Science 277, 968-971.

Rainville, P., Hofbauer, R.K., Paus, T., Duncan, G.H., Bushnell, M.C., Price, D.D., 1999. Cerebral mechanisms of hypnotic induction and suggestion. J. Cogn. Neurosci. 11 (1), 110-125.

Robbins, T.W., 1997. Arousal systems and attentional processes. Biol. Psychol. 45 (1-3), 57-71.

Shor, R.E., Orne, E.C., 1962. The Harvard group scale of hypnotic susceptibility, form A. Consulting Psychologists Press, Palo Alto, CA.

Spencer, K.M., Dien, J., Donchin, E., 1999. A componential analysis of the ERP elicited by novel events using a dense electrode array. Psychophysiology 36 (3), 409-414.

Spiegel, D., Albert, L.H., 1983. Naloxone fails to reverse hypnotic alleviation of chronic pain. Psychopharmacology 81 (2), 140-143.

Spiegel, D., Cutcomb, S., Ren, C., Pribram, K., 1985. Hypnotic hallucination alters evoked potentials. J. Abnorm. Psychol. 94 (3), 249-255.

Watanabe, S., Kakigi, R., Koyama, S., Hoshiyama, M., Kaneoke, Y., 1998. Pain processing traced by magnetoencephalography in the human brain. Brain Topogr. 10, 255-264.

Weitzenhoffer, A.M., Hilgard, E.R., 1962. Stanford hypnotic susceptibility scale: form C. Consulting Psychologist Press, Palo Alto, CA.

Wijers, A.A., Lange, J.J., Mulder, G., Mulder, L.J., 1997. An ERP study of visual spatial attention and letter target detection for isoluminant and nonisoluminant stimuli. Psychophysiology 34 (5), $553-$ 565.

Yamasaki, H., Kakigi, R., Watanabe, S., Naka, D., 1999. Effects of distraction on pain perception: magneto- and electro- encephalographic studies. Brain Res. Cogn. Brain Res. 8 (1), 73-76. 